\title{
A Fast-Track Program Reduces Complications and Length of Hospital Stay After Open Colonic Surgery
}

\author{
SVEN MULLER, ${ }^{*, \neq}$ MARCO P. ZALUNARDO, ${ }^{\S}$ MARTIN HUBNER, ${ }^{\ddagger}$ PIERRE A. CLAVIEN, ${ }^{*}$ NICOLAS DEMARTINES, ${ }^{*, \ddagger}$ and \\ the Zurich Fast Track Study Group
}

*Visceral and Transplant Surgery, and the \$Institute of Anesthesiology, University Hospital, Zurich, Switzerland; and the ₹Department of Visceral Surgery, Centre Hospitalier Universitaire Vaudois Lausanne, Lausanne, Switzerland

See related article, Nguyen GC et al, on page 329 in $\mathrm{CGH}$.

Background \& Aims: A fast-track program is a multimodal approach for patients undergoing colonic surgery that combines stringent regimens of perioperative care (fluid restriction, optimized analgesia, forced mobilization, and early oral feeding) to reduce perioperative morbidity, hospital stay, and cost. We investigated the impact of a fast-track protocol on postoperative morbidity in patients after open colonic surgery. Methods: A randomized trial of patients in $\mathbf{4}$ teaching hospitals in Switzerland included 156 patients undergoing elective open colonic surgery who were assigned to either a fast-track program or standard care. The primary end point was the 30-day complication rate. Secondary end points were severity of complications, hospital stay, and compliance with the fast-track protocol. Results: The fast-track protocol significantly decreased the number of complications (16 of 76 in the fast-track group vs 37 of 75 in the standard care group; $P=.0014)$, resulting in shorter hospital stays (median, 5 days; range, 2-30 vs 9 days, respectively; range, $6-30 ; P<.0001)$. There was a trend toward less severe complications in the fast-track group. A multiple logistic regression analysis revealed fluid administration greater than the restriction limits (odds ratio, $4.198 ; 95 \%$ confidence interval, 1.7-10.366; $P=.002)$ and a nonfunctioning epidural analgesia (odds ratio, $3.365 ; 95 \%$ confidence interval, 1.367-8.283; $P=.008$ ) as independent predictors of postoperative complications. Conclusions: The fast-track program reduces the rate of postoperative complications and length of hospital stay and should be considered as standard care. Fluid restriction and an effective epidural analgesia are the key factors that determine outcome of the fast-track program.

$F_{d}$ ast-track programs in colonic surgery were introduced to reduce postoperative morbidity, shorten hospital stay, improve patient convenience, and decrease costs. The main objective of this multimodal concept mostly focused on perioperative fluid restriction, an optimized analgesic regimen, a forced mobilization, and early postoperative oral nutrition. ${ }^{1-3}$ Cohort studies and small randomized studies have shown that fast-track concepts reduce hospital stay and morbidity, and may improve postoperative pulmonary function and fatigue tolerance. ${ }^{4-10}$ Few controlled trials with an adequate number of patients comparing the current standard approach with the fast track concept showing the superiority of this method in colonic surgery in regard to a reduced complication rate exists. Therefore, we conducted a multicentric, randomized, controlled trial to investigate the impact of a fast-track protocol on postoperative morbidity in open colonic surgery. As secondary end points we studied the severity of complications according to a validated classification score ${ }^{11}$ the length of hospital stay, and the compliance to the different components of the fast-track program.

\section{Patients and Methods \\ Patients and Eligibility}

We conducted a multicenter, randomized trial in 4 surgical departments (teaching hospitals) in Switzerland. Eligible for this trial were patients older than 18 years of age who were undergoing open elective colonic resection with a primary anastomosis. Exclusion criteria were emergency situations, contraindication to epidural anesthesia, scheduled total colectomy or rectum resection, and preoperatively immobile patients. The study was approved by the local ethical committees of the participating hospitals according to the Declaration of Helsinki, ${ }^{12}$ and all patients provided written informed consent. From November 2004 until October 2006 there were 156 patients randomized online via a commercial software program (Randomizer; Institute for Medical Informatics, Statistics and Documentation, Medical University of Graz, Austria; available at: http://www. randomizer.at/) to either standard care or the fasttrack program. The randomization was stratified for 
the center. Blinding of the patients and investigators was not possible because of the differences in interventional regimens.

\section{Study Design}

A slightly adapted fast-track protocol from the one proposed by Kehlet and Wilmore ${ }^{1}$ was used. In both groups no bowel preparation was performed and patients were allowed to drink clear fluids until 4 hours before surgery. All patients received thromboprophylaxis and perioperative antibiotics. Surgical experience was guaranteed by at least one specialized surgeon in colorectal surgery attending the procedure. Surgery was performed through a median laparotomy and the anastomosis was either hand sewn or a stapler technique was used. No nasogastric tubes or intra-abdominal drains were used postoperatively. All anesthetic procedures and agents were standardized. Patients in the fasttrack group received a restricted fluid regimen with a preoperative loading of Ringer's lactate solution at $1 \mathrm{~mL} /$ $\mathrm{kg} / \mathrm{h}$ nothing by mouth and an intraoperative substitution of $5 \mathrm{~mL} / \mathrm{kg} / \mathrm{h}$. The standard care group received Ringer's lactate at $2 \mathrm{~mL}$ and $10 \mathrm{~mL} / \mathrm{kg} / \mathrm{h}$ for preoperative loading and intraoperative substitution, respectively. All fluids in the fast-track group were discontinued at day 1 after surgery unless there was a medical reason to do otherwise. In the standard care group the patients received $2000 \mathrm{~mL}$ of Ringer's lactate per 24 hours until day 3 after surgery. Additional fluid or vasopressors were given when the mean arterial pressure was less than $60 \mathrm{~mm} \mathrm{Hg}$ or urine output was less than $0.5 \mathrm{~mL} / \mathrm{kg} / \mathrm{h}$. The transfusion limit was a hematocrit level less than $25 \%$. All patients were encouraged to early mobilization starting immediately after surgery in both groups. In the fast-track group, patients were allowed to start drinking immediately after surgery. Two additional protein drinks were given (Fresenius Power Drink; Fresenius Kabi, Stans, Switzerland) for the first 3 days, and patients were invited to resume oral nutrition on day 1 after surgery. Patients in the standard care group were allowed to start drinking on day 2 and started increasing oral nutrition on day 2 , with possible full oral nutrition by day 4 . Criteria for reinsertion of a nasogastric tube were 2 consecutive episodes of vomiting greater than $400 \mathrm{~mL}$.

An epidural catheter with ropivacaine $0.33 \%$ or bupivacaine $0.25 \%$ was placed at thoracic level 6-9 preoperatively and removed on the second postoperative day. As additional analgesics, only paracetamol was given intravenously. A failure of epidural analgesia was defined as the need for supplemental intravenous opioids. Discharge from the hospital was suggested when patients were fully mobile, pain was controlled by oral analgesics only, tolerance of oral food was established, and the patients felt comfortable with a discharge. Oral food intake was considered sufficient if the patient was able to eat more than two thirds of the daily meal.

\section{Outcomes and Follow-Up Evaluation}

The primary end point of this study was total complications occurring until 30 days after surgery. All complications were recorded until 30 days after surgery with telephone follow-up evaluation if necessary. Complications were classified in general or as surgical complications. For grading the severity of complications a validated complication classification was used. ${ }^{11}$ The total intraoperative (including preoperative loading and fluid administered intraoperatively) and the first 24 hours postoperative fluid administration were recorded. Oral food intake and mobilization time was documented via a patient self-assessment sheet.

\section{Sample Size Calculation and Statistical Analysis}

The calculated sample size was based on the assumption of previous local experience that a complication rate of $30 \%$ in the standard care group would be reduced to $20 \%$ in the fast-track group. To achieve an $80 \%$ power with a 2 -sided $P$ value of less than .05 as significant, 231 patients per group were required. An interim analysis was planned after inclusion of one third of the calculated sample size with a premature study end, if any difference in the primary end point according to the $\mathrm{O}^{\prime}$ Brien-Fleming ${ }^{13}$ approach was reached $(P<.0019)$. Statistical analysis was based on an intention-to-treat analysis and was performed with the Statistical Package for Social Science software (version 14.0; SPSS, Chicago, IL). The Mann-Whitney $U$ test was used to compare continuous variables, the chi-square test and the Fisher exact test were used to compare discrete variables. The factors of blood loss greater than $500 \mathrm{~mL}$, need for blood transfusion, type of surgery, age older than 70 years, body mass index greater than $30 \mathrm{~kg} / \mathrm{m}^{2}$, preoperative hematocrit level, functioning EDA, American Society of Anesthetists class higher than III, fluid restriction limits, removal of the urinary catheter after 24 hours, and successful oral nutrition at days 1 and 4, were analyzed in a univariate logistic regression analysis if they were associated with postoperative complications. Factors identified as significant in a univariate logistic regression analysis were selected to perform a multiple logistic regression analysis.

\section{Results}

From November 2004 until October 2006 there were 156 patients who fulfilled inclusion criteria and were assigned randomly to the fast-track and standard care groups. A planned interim analysis was performed after inclusion of one third of the calculated sample size and the study was stopped prematurely after reaching a significant difference for the primary end point according to the O'Brien-Fleming approach. Thus, 151 patients completed the study period (supplementary Table 1; see supplementary material online at www.gastrojournal.org). 
Table 1. Patient Characteristics and Surgical Details

\begin{tabular}{|c|c|c|c|}
\hline & $\begin{array}{c}\text { Fast-track } \\
\mathrm{n}=76\end{array}$ & $\begin{array}{l}\text { Control } \\
\mathrm{n}=75\end{array}$ & $P$ value \\
\hline Median age, $y$ (range) & $62(27-91)$ & $59(39-89)$ & .04 \\
\hline Male/female & $37 / 39$ & $40 / 35$ & .63 \\
\hline Malignant/benign & $67 / 9$ & $64 / 11$ & .64 \\
\hline \multicolumn{4}{|l|}{ ASA score } \\
\hline 1 & 2 & 3 & .55 \\
\hline II & 50 & 54 & \\
\hline III & 24 & 18 & \\
\hline Median body mass index, $\mathrm{kg} / \mathrm{m}^{2}$ (range) & $24(19-35)$ & $26(17-33)$ & .63 \\
\hline \multicolumn{4}{|l|}{ Type of surgery } \\
\hline Sigmoid resection or left hemicolectomy & 50 & 51 & .31 \\
\hline Resection of transverse colon & 0 & 2 & \\
\hline Right hemicolectomy & 26 & 22 & \\
\hline Median blood loss, $m L$ (range) & $260(0-2100)$ & $320(0-1350)$ & .85 \\
\hline Median duration of surgery, min (range) & $140(60-255)$ & $120(55-285)$ & 11 \\
\hline
\end{tabular}

ASA, American Society of Anesthetists.

The relevant patient characteristics and surgical details are shown in Table 1, disclosing a well-balanced distribution of patients between the groups, with the exception of age showing a slight difference (62 years in the fast track vs 59 years in the standard group).

In the intent-to-treat analysis, the primary end point of total complications occurring during the first 30 days after surgery was significantly lower in the fast-track group compared with the standard care group (16 of 76 vs 37 of $75 ; P=.0014)$. This was mainly owing to a reduction in general complications, whereas surgical complications were reduced in the fast-track group. There was also a trend towards more severe complications in the standard care group compared with the fast-track group using a standard classification of complications by severity. The median hospital stay was 5 days (range, 2-30 days) in the fast-track group vs 9 days (range, 6-30 days) in the standard care group $(P<$ $.0001)$. No significant difference in the re-admission rate ( 2 and 3 patients) was observed between the groups until 30 days after surgery (Table 2 ). There was no significant difference in anastomotic insufficiencies ( 1 in the fasttrack group and 2 in the standard care group). A list of the types of complications is available in supplementary Table 1 (see supplementary material online at www. gastrojournal.org).

Adherence to the fast-track and standard care protocols is summarized in Table 3 , showing excellent compliance. There were no severe adverse events observed as a result of the EDA (infection or epidural hematoma). Rates of re-insertion of a nasogastric tube for recurrent vomiting were not different, with 3 patients in the fasttrack group vs 2 patients in the standard care group. No significant changes in preoperative and postoperative serum creatinine levels were observed in both groups (median delta creatinine level of $4 \mu \mathrm{mol} / \mathrm{L}$ in the fast track group vs $1 \mu \mathrm{mol} / \mathrm{L}$ in the standard care group; $P=.29$ ).
In a multiple logistic regression analysis, only a failed EDA and administration of fluid greater than the restriction limits were the significant independent predictors for postoperative complications (Table 4).

In a second analysis, fast-track patients who fulfilled the fluid restriction criteria and had an effective EDA were included ( $\mathrm{n}=59$ of 76 ). This subgroup had an even lower complication rate of only $7 \%$, and a median hospital stay of only 4 days (range, 2-20 days).

In contrast, the 17 patients in whom the fluid restriction and a functioning EDA were not applied completely had similar results to the patients in the standard care group regarding both the complication rate $(47 \%)$ and the hospital stay (12 days).

\section{Discussion}

The results of this randomized multicenter trial indicate that a fast-track program significantly reduces

Table 2. Results: Primary and Secondary End Points

\begin{tabular}{|c|c|c|c|}
\hline & $\begin{array}{l}\text { Fast-track } \\
(\mathrm{n}=76)\end{array}$ & $\begin{array}{l}\text { Control } \\
(n=75)\end{array}$ & $P$ value \\
\hline Total complications $^{a}$ & 16 & 37 & .0014 \\
\hline General complications & 7 & 21 & .006 \\
\hline Surgical complications & 9 & 16 & .13 \\
\hline Patients with a complication ${ }^{b}$ & $13(17 \%)$ & $28(37 \%)$ & .006 \\
\hline Grade I & 5 & 10 & \\
\hline Grade II & 5 & 11 & \\
\hline Grade IIla & 0 & 0 & \\
\hline Grade IIIb & 1 & 2 & \\
\hline Grade IVa & 1 & 2 & \\
\hline Grade IVb & 0 & 2 & \\
\hline Grade V & 1 & 1 & \\
\hline Rehospitalization & 3 & 2 & 1 \\
\hline Median hospital stay (range) & $5(2-30)$ & $9(6-30)$ & $<.0001$ \\
\hline
\end{tabular}

aMore than 1 complication was counted per patient. ${ }^{b}$ Severity of complications classification, ${ }^{11}$ counting the severest complication per patient. 
Table 3. Adherence to the Study Protocols

\begin{tabular}{|c|c|c|c|}
\hline & $\begin{array}{c}\text { Fast-track } \\
(\mathrm{n}=76)\end{array}$ & $\begin{array}{c}\text { Control } \\
(n=75)\end{array}$ & $P$ value \\
\hline Median intraoperative intravenous intake, $m L$ (range) & $1925(1100-6700)$ & $2950(1600-6400)$ & $<.0001$ \\
\hline First 24-hour median intravenous intake, $m L$ (range) & $2700(1800-9100)$ & $5200(3900-9900)$ & $<.0001$ \\
\hline Effective EDA, $n$ & $61(80 \%)$ & $59(79 \%)$ & .84 \\
\hline Median mobilization time day $1, \min$ (range) & $240(0-480)$ & $240(0-480)$ & .48 \\
\hline$>2 / 3$ oral nutrition day 1 & $38 \%$ & 0 & \\
\hline$>2 / 3$ oral nutrition day 4 & $93 \%$ & $63 \%$ & $<.0001$ \\
\hline
\end{tabular}

the number of postoperative complications, especially general complications, leading to a shorter hospital stay after elective open colonic surgery. In a multivariate analysis, the present study shows that intraoperative fluid restriction and an effective EDA are significant predictors of reduced postoperative complications. Methodologic limitations of this study, which may cause a bias of either study group, were that blinding of patients and surgeons was not possible because of the obvious different fluid regimens and resumption of oral nutrition between the 2 groups.

Our trial was initiated to yield convincing evaluation of a fast-track program in the daily practice of open colonic surgery. Previously published cohort studies and underpowered randomized controlled trials have suggested that fast-track programs offer benefits regarding the duration of hospitalization, hospital stay, morbidity, and metabolic and functional results; for example, pulmonary function and bowel transit were better when patients were included in a fast-track protocol. ${ }^{2,4,10} \mathrm{~A}$ recently published European survey showed that in clinical practice most colonic surgery is still performed in a traditional manner, with a hospital stay exceeding 10 days. ${ }^{14}$

In the present trial an effective EDA and a stringent fluid regimen were the only 2 independent factors associated with a significant reduction of complications. These results were enhanced further with the subgroup analysis of patients who completely fulfilled the protocol. Interestingly, successful early postoperative nutrition was not correlated with a reduced complication rate. A delay in resuming postoperative oral nutrition seems to be an indicator of failed integral fast-track components such as fluid restriction and EDA.

Existing evidence on the benefits of EDA in colonic surgery with or without a fast track program have been well studied ${ }^{15-17}$ including a meta-analysis by Marret et al. ${ }^{18}$ In our study a failed EDA appeared to inhibit the success of a fast-track program including especially enforced mobilization and early nutrition. Perioperative fluid restriction in colonic surgery was shown to reduce morbidity and length of hospital stay in fast-track protocols in 2 randomized controlled trials. ${ }^{19,20}$ In contrast, in another trial with postoperative fluid restriction and a fast-track protocol no benefit on morbidity and the length of hospitalization could be shown. ${ }^{21}$ This might be because the difference of fluid amount administered on the day of surgery probably was too small, with less than 1-L difference between the groups. Therefore, our results and the findings of the 2 trials mentioned before ${ }^{19,20}$ convincingly show that a restricted fluid regimen during surgery and in the early postoperative days is an important factor of outcome improvement.

A major concern about fast-track programs with early discharge may be the risk of missing severe postoperative complications. Pioneer works on fast-track concepts reported a very short hospital stay of only 2 days, but then observed a significantly higher re-admission rate. ${ }^{4}$ In the present study the re-admission rate was low and similar between the 2 groups. Furthermore, patients were observed for up to 30 days postoperatively, which considerably lessens the risk of missing a complication. In patients in fast-track programs with a slightly prolonged minimal hospital stay of up to 3 days, no higher readmission rates were observed. ${ }^{22}$

A further important issue is the role of fast-track programs regarding the increasing use of laparoscopic colon surgery. Large randomized trials comparing open vs laparoscopic surgery showed significant benefits in length of hospital stay, analgesia, and overall patient comfort for laparoscopic procedures. ${ }^{23,24}$ However, these trials failed to disclose a standardized fast-track regimen. In a recently published randomized study, a fast-track

Table 4. Results of Multiple Logistic Regression Analysis

\begin{tabular}{lccc}
\hline & \multicolumn{3}{c}{ Postoperative complications } \\
\cline { 2 - 4 } & P value & Odds ratio & $95 \% \mathrm{Cl}$ \\
\hline Above the limits of fluid restriction vs within limits & .002 & 4.198 & $1.700-10.366$ \\
Failed vs effective EDA & .008 & 3.365 & $1.367-8.283$ \\
Nutrition day $4:>2 / 3$ not achieved vs achieved & .971 & 0.983 & $0.385-2.505$ \\
Blood loss $>500$ vs $<500 \mathrm{~mL}$ & .204 & 1.866 & $.713-4.882$ \\
\hline
\end{tabular}


program in open surgery compared with laparoscopic surgery without a standardized fast track concept disclosed a similar length of hospital stay and complication rates. ${ }^{25}$

In conclusion, our findings show that a fast-track program is beneficial in terms of reducing morbidity and length of hospital stay, in patients undergoing elective colon surgery, provided that an effective EDA and a stringent perioperative fluid restriction are used.

\section{Investigators of the Zurich Fast Track Trial Group}

Sven Muller, MD, and Nicolas Demartines, MD, Department of Visceral Surgery, CHUV, Lausanne, and formerly Visceral and Transplant Surgery, University Hospital Zurich; Pierre A. Clavien, MD, PhD, Visceral and Transplant Surgery, University Hospital Zurich; Marco P. Zalunardo, MD, Institute of Anesthesiology, University Hospital Zurich; Marco Decurtins, MD, Department of Surgery, Kantonsspital Winterthur; Lukas Eisner, MD, Department of Surgery, Kantonsspital Olten; Italo Castelli, MD, Department of Anesthesiology, Kantonsspital Olten; Hans P. Keller, MD, Department of Anesthesiology, Kantonsspital Winterthur; Martin Hubner, MD, and Igor Langer, MD, Department of Visceral Surgery, CHUV, Lausanne; Hans Gelpke, MD, and Felix Grieder, MD, Department of Surgery, Kantonsspital Winterthur; Thorsten Carstensen, MD, and Andreas Gehrz, MD, Department of Surgery, Kantonsspital Olten; Thierry Spahr, $\mathrm{MD}$, Institute of Anesthesiology, University Hospital Zurich; and Reto Paganoni, MD, Department of Anesthesiology, Kantonsspital Olten.

\section{Supplementary Data}

Note: To access the supplementary material accompanying this article, visit the online version of Gastroenterology at www.gastrojournal.org, and at doi: 10.1053/j.gastro.2008.10.030.

\section{References}

1. Kehlet $\mathrm{H}$, Wilmore DW. Multimodal strategies to improve surgical outcome. Am J Surg 2002;183:630-641.

2. Wind J, Polle SW, Fung Kon Jin PH, et al. Systematic review of enhanced recovery programmes in colonic surgery. $\mathrm{Br} J$ Surg 2006;93:800-809.

3. Fearon KC, Ljungkvist $\mathrm{O}$, Von Meyenfeldt M, et al. Enhanced recovery after surgery: a consensus review of clinical care for patients undergoing colonic resection. Clin Nutr 2005;24:466477.

4. Basse L, Thorbol JE, LossI K, et al. Colonic surgery with accelerated rehabilitation or conventional care. Dis Colon Rectum 2004; 47:271-278.

5. Bradshaw BG, Liu SS, Thirlby RC. Standardized perioperative care protocols and reduced length of stay after colon surgery. J Am Coll Surg 1998;186:501-506.

6. Delaney CP, Zutshi M, Senagore AJ, et al. Prospective, randomized, controlled trial between a pathway of controlled rehabilitation with early ambulation and diet and traditional postoperative care after laparotomy and intestinal resection. Dis Colon Rectum 2003;46:851-859.

7. DiFronzo LA, Yamin N, Patel K, et al. Benefits of early feeding and early hospital discharge in elderly patients undergoing open colon resection. J Am Coll Surg 2003;197:747-752.

8. Anderson AD, McNaught CE, MacFie J, et al. Randomized clinical trial of multimodal optimization and standard perioperative surgical care. Br J Surg 2003;90:1497-1504.

9. Gatt M, Anderson AD, Reddy BS, et al. Randomized clinical trial of multimodal optimization of surgical care in patients undergoing major colonic resection. Br J Surg 2005;92:1354-1362.

10. Khoo CK Vickery $\mathrm{CJ}$, Forsyth $\mathrm{N}$, et al. A prospective randomized controlled trial of multimodal perioperative management protocol in patients undergoing elective colorectal resection for cancer. Ann Surg 2007;245:867-872.

11. Dindo D, Demartines N, Clavien PA. Classification of surgical complications: a new proposal with evaluation in a cohort of 6336 patients and results of a survey. Ann Surg 2004;240:205-213.

12. Blackmer JHH. The Declaration of Helsinki: an update on paragraph 30. CMAJ 2005;173:1052-1053.

13. Schulz KF, Grimes DA. Multiplicity in randomised trials II: subgroup and interim analysis. Lancet 2005;365:1657-1661.

14. Kehlet $\mathrm{H}$, Buchler MW, Beart RW Jr, et al. Care after colonic operation-is it evidence-based? Results from a multinational survey in Europe and the United States. J Am Coll Surg 2006; 202:45-54.

15. Carli F, Mayo N, Klubien K, et al. Epidural analgesia enhances functional exercise capacity and health-related quality of life after colonic surgery: results of a randomized trial. Anesthesiology 2002;97:540-549.

16. Steinberg RB, Liu SS, Wu CL, et al. Comparison of ropivacainefentanyl patient-controlled epidural analgesia with morphine intravenous patient-controlled analgesia for perioperative analgesia and recovery after open colon surgery. J Clin Anesth 2002 14:571-577.

17. Taqi A, Hong X, Mistraletti G, et al. Thoracic epidural analgesia facilitates the restoration of bowel function and dietary intake in patients undergoing laparoscopic colon resection using a traditional, nonaccelerated, perioperative care program. Surg Endosc 2007;21:247-252.

18. Marret E, Remy C, Bonnet F. Meta-analysis of epidural analgesia versus parenteral opioid analgesia after colorectal surgery. $\mathrm{Br} J$ Surg 2007;94:665-673.

19. Brandstrup B, Tonnesen H, Beier-Holgersen R, et al. Effects of intravenous fluid restriction on postoperative complications: comparison of two perioperative fluid regimens: a randomized assessorblinded multicenter trial. Ann Surg 2003;238:641-648.

20. Lobo DN, Bostock KA, Neal KR, et al. Effect of salt and water balance on recovery of gastrointestinal function after elective colonic resection: a randomised controlled trial. Lancet 2002; 359:1812-1818.

21. MacKay G, Fearon K, McConnachie A, et al. Randomized clinical trial of the effect of postoperative intravenous fluid restriction on recovery after elective colorectal surgery. Br J Surg 2006;93: 1469-1474.

22. Andersen JH-JD, Christiansen PS, Kehlet H. Readmission rates after a planned hospital stay of 2 versus 3 days in fast-track colonic surgery. Br J Surg 2007;94:890-893.

23. A comparison of laparoscopically assisted and open colectomy for colon cancer. N Engl J Med 2004;350:2050-2059.

24. Veldkamp R Kuhry E, Hop WC, et al. Laparoscopic surgery versus open surgery for colon cancer: short-term outcomes of a randomised trial. Lancet Oncol 2005;6:477-484.

25. Basse L, Jakobsen DH, Bardram L, et al. Functional recovery after open versus laparoscopic colonic resection: a random ized, blinded study. Ann Surg 2005;241:416-423. 
Received July 18, 2008. Accepted October 16, 2008.

\section{Reprint requests}

Address requests for reprints to: Nicolas Demartines, MD,

Professor and Chairman, Department of Visceral Surgery, University Hospital CHUV, Rue de Bugnon 46, CH-1011 Lausanne, Lausanne, Switzerland. e-mail: demartines@chuv.ch; fax: 0041-21-314-2411.

\section{Acknowledgements}

Members of the Zurich Fast Track Study Group are listed at the end of the article.

Trial Registration: http://www.clinicaltrials.gov; identifier: NCT00556790.
Dr Muller designed the study, analyzed the data, performed statistical analysis and drafted the manuscript and had full access to all of the data in the study and takes responsibility for the integrity of the data and the accuracy of the data analysis. Dr Zalunardo, Dr Hubner, and Dr Clavien participated in the design and coordination of the study and data analyses and helped to draft the manuscript. Dr Demartines designed the study, analyzed the data, and helped to draft the manuscript. All authors read and approved the final manuscript.

\section{Conflicts of interest}

The authors disclose the following: Dr Muller, Dr Clavien, and Dr Demartines have received research support from Fresenius Kabi, Stans, Switzerland. Dr Zalunardo and Dr Hubner disclose no conflicts. 
Supplementary Table 1. Types of Complications

\begin{tabular}{lcc}
\hline & $\begin{array}{c}\text { Fast-track } \\
\mathrm{n}=76\end{array}$ & $\begin{array}{c}\text { Control } \\
\mathrm{n}=75\end{array}$ \\
\hline Intra-abdominal abscess & 0 & 1 \\
Wound infection & 4 & 7 \\
Anastomotic leaks & 1 & 2 \\
Cardiovascular events & 3 & 9 \\
Urinary infection/retention & 3 & 5 \\
Postoperative bleeding & 1 & 2 \\
Pneumonia/respiratory events & 1 & 4 \\
Postoperative ileus & 3 & 4 \\
Other events & 0 & 3 \\
\hline
\end{tabular}

